PROCEEDINGS OF THE

AMERICAN MATHEMATICAL SOCIETY

Volume 129, Number 1, Pages 265-270

S 0002-9939(00)05499-X

Article electronically published on July 27, 2000

\title{
STRONGLY MEAGER SETS AND THEIR UNIFORMLY CONTINUOUS IMAGES
}

\author{
ANDRZEJ NOWIK AND TOMASZ WEISS \\ (Communicated by Carl G. Jockusch, Jr.)
}

\begin{abstract}
We prove the following theorems:
(1) Suppose that $f: 2^{\omega} \rightarrow 2^{\omega}$ is a continuous function and $X$ is a Sierpiński set. Then

(A) for any strongly measure zero set $Y$, the image $f[X+Y]$ is an $s_{0}$-set,

(B) $f[X]$ is a perfectly meager set in the transitive sense.
\end{abstract}

(2) Every strongly meager set is completely Ramsey null.

This paper is a continuation of earlier works by the authors and by M. Scheepers (see [N], NSW], [S]) in which properties (mainly, the algebraic sum) of certain singular subsets of the real line $\mathbf{R}$ and of the Cantor set $2^{\omega}$ were investigated. Throughout the paper, by a set of real numbers we mean a subset of $2^{\omega}$ and by "+" we denote the standard modulo 2 coordinatewise addition in $2^{\omega}$. Let us also assume that a "measure zero" (or "negligible") set always denotes a Lebesgue measure zero set. We apply the following definition of sets of real numbers.

Definition 1. An uncountable set $X$ is said to be a Luzin (respectively, Sierpiński) set iff for each meager (respectively, measure zero) set $Y, X \cap Y$ is at most countable. We say that a set $X$ is of strong measure zero (respectively, strongly meager) iff for each meager (respectively, measure zero) set $Y, X+Y \neq 2^{\bar{\omega}}$.

Remark 1. It is well known (see [M] for example) that every Luzin set is strongly measure zero. Quite recently J. Pawlikowski proved that each Sierpiński set must be strongly meager as well (see $[\mathrm{P}]$ ). Let us recall that a set $X$ is called an $s_{0}$-set (or Marczewski set) iff for each perfect set $P$ one can find a perfect set $Q \subseteq \overline{P \text { that }}$ is disjoint from $X$. M. Scheepers showed in $[\mathrm{S}]$ that for a Sierpiński set $X$ and a strong measure zero set $Y, X+Y$ is an $s_{0}$-set. Later, in [NSW] it was proven that this also holds when $X$ is strongly meager. We have the following functional version of the M. Scheepers' result.

Theorem 1. Let $X$ be a Sierpiński set and let $Y$ be a strong measure zero set. Assume also that $f: 2^{\omega} \rightarrow 2^{\omega}$ is a continuous function. Then the image $f[X+Y]$ is an $s_{0}$-set.

Received by the editors July 16, 1998 and, in revised form, September 9, 1998 and March 10, 1999.

2000 Mathematics Subject Classification. Primary 03E15, 03E20, 28 E15.

Key words and phrases. Strongly meager set, always first category set.

The first author was partially supported by the KBN grant 2 P03A 04709.

(C)2000 American Mathematical Society 
Proof. Let $P \subseteq 2^{\omega}$ be a perfect set. We can assume that $f\left[2^{\omega}\right] \cap P$ contains a perfect set. Otherwise, we are done. So, fix $\left\{R_{\alpha}: \alpha<\omega_{1}\right\}$, a family of pairwise disjoint perfect subsets contained in $f\left[2^{\omega}\right] \cap P$, and for every $\alpha<\omega_{1}$, put $R_{\alpha}^{\prime}=f^{-1}\left[R_{\alpha}\right]$. Take $\alpha_{0}<\omega_{1}$ such that $R_{\alpha_{0}}^{\prime} \in \mathcal{N}$ (negligible sets). We know that $Y$ is of strong measure zero and that $R_{\alpha_{0}}^{\prime}$ is closed, so $R_{\alpha_{0}}^{\prime}-Y$ has measure zero. From this we have that $X \cap\left(R_{\alpha_{0}}^{\prime}-Y\right)$ (let us denote this set by $\left.X^{\prime}\right)$ is countable. Thus,

$$
f[X+Y]=f\left[\left(X \backslash X^{\prime}\right)+Y\right] \cup f\left[X^{\prime}+Y\right]
$$

and $f\left[\left(X \backslash X^{\prime}\right)+Y\right]$ is disjoint from $R_{\alpha_{0}}$. Also, since $f$ is a uniformly continuous function, $f\left[X^{\prime}+Y\right]$ is a strong measure zero set. Hence $f[X+Y]$ is disjoint from some perfect set contained in $R_{\alpha_{0}}$.

Definition 2. A set $X$ is called an $\underline{\mathrm{AFC}}$ ' set (perfectly meager in the transitive sense) iff for each perfect set $P$ there is $F$, an $F_{\sigma}$ set containing $X$, such that for every $t \in 2^{\omega},(F+t) \cap P$ is meager in the relative topology of $P$. We will say that $X$ is a wQN-set (weakly Quasinormal set) iff for each sequence of continuous functions $f_{n} \overline{: X} \rightarrow \mathbf{R}$, if $f_{n} \rightarrow 0$ (pointwise), then there is a subsequence $f_{n_{k}}$ and countable family $\left\{X_{n}\right\}_{n \in \omega}$ such that $X=\bigcup_{n \in \omega} X_{n}$ and $f_{n_{k}}$ converges uniformly on $X_{n}$ for every $n \in \omega$.

It is easy to prove that each Sierpiński set is wQN and that for a wQN-set $X$ and every continuous function $f: 2^{\omega} \rightarrow 2^{\omega}, f[X]$ is a wQN-set as well (see [BRR]). Thus, using Nowik's theorem which says that any wQN-set is an AFC' set (see [N]), we obtain the following theorem.

Theorem 2. If $S$ is a Sierpinski set, then for every continuous function $f: 2^{\omega} \rightarrow$ $2^{\omega}$, we have that $f[S]$ is an $A F C^{\prime}$ set.

We present an alternative proof of this fact with the hope that it may lead to a positive answer to Question 1 (see below).

Lemma 1 (Nowik). For each perfect set $P \subseteq 2^{\omega}$, there exists a continuous function $\Phi: 2^{\omega} \rightarrow 2^{\omega}$ such that for any $t \in 2^{\omega}, \Phi[P+t]=2^{\omega}$.

Proof. See $[\mathrm{N}]$.

Corollary 1. Let $P \subseteq 2^{\omega}$ be a perfect set. Then there exists an uncountable family $\mathcal{H}$ of pairwise disjoint closed subsets of $2^{\omega}$ such that for every $G \in \mathcal{H}, P+G=2^{\omega}$.

Proof. Let $\Phi$ be as in Lemma 1, Put $\mathcal{H}=\left\{\Phi^{-1}[\{h\}]: h \in 2^{\omega}\right\}$.

Corollary 2. For every perfect set $P \subseteq 2^{\omega}$, there exists an uncountable family $\mathcal{H}$ of closed, pairwise disjoint negligible sets such that for each $G \in \mathcal{H}, P+G=2^{\omega}$.

Proof. Obvious, since for $\mathcal{H}$ in Corollary 1 we have that $|\{G \in \mathcal{H}: G \notin \mathcal{N}\}| \leq$ $\omega$.

Proof of Theorem 圆 Let $P \subseteq 2^{\omega}$ be a perfect set and let $f$ be a continuous function. Without loss of generality we may assume that $f$ maps $2^{\omega}$ onto $2^{\omega}$. Suppose that $\left(P_{i}\right)_{i \in \omega}$ is an enumeration of basic clopen sets in the relative topology of $P$. Assume that for each $i \in \omega, \mathcal{H}^{i}$ is an uncountable family of pairwise disjoint, closed sets such that

Let

$$
\forall_{G \in \mathcal{H}^{i}} P_{i}+G=2^{\omega} .
$$

$$
\tilde{\mathcal{H}}^{i}=\left\{f^{-1}[G]: G \in \mathcal{H}^{i}\right\}
$$


We choose from every $\tilde{\mathcal{H}}^{i}$ a negligible set $A_{i}$. Suppose that $B$, a $G_{\delta}$ negligible set, is such that

$$
\bigcup_{i<\omega} A_{i} \subseteq B
$$

and $C=2^{\omega} \backslash B$. Since $S$ is a Sierpiński set, $S^{\prime}=S \cap B$ is at most countable. We have that

$$
f[C] \cap \bigcup_{i<\omega} f\left[A_{i}\right]=\emptyset
$$

so

$$
f\left[S \backslash S^{\prime}\right] \cap \bigcup_{i<\omega} f\left[A_{i}\right]=\emptyset .
$$

It is clear that $f[C]$ is an $F_{\sigma}$ set; thus $f\left[S \backslash S^{\prime}\right]$ is disjoint from some $G_{\delta}$ set $A$ which contains $\bigcup_{i<\omega} f\left[A_{i}\right]$. Finally, for every $t \in 2^{\omega}, f\left[S \backslash S^{\prime}\right] \cap(P-t)$ is disjoint from $A \cap(P-t)$. From the fact that $f\left[A_{i}\right]+P_{i}=2^{\omega}$ (for every $i<\omega$ ) it follows that $A$ is a dense set in $P-t$.

Remark 2. Notice that Corollary 2 is a stronger version of the well-known ErdösKunen-Mauldin theorem (see [NSW]).

Definition 3. For any finite set $s \in[\omega]^{<\omega}$ and infinite $A \subseteq \omega$ with $\max (s)<$ $\min (A)$, let $[s, A]=\left\{B \in[\omega]^{\omega}: s \subseteq B \subseteq s \cup A\right\}$. We say that $F \subseteq[\omega]^{\omega}$ is a completely Ramsey null $\left(C R_{0}\right)$ set iff for every so-called Ellentuck basic neighbourhood $[s, A]$, there is $B \subseteq A$ infinite such that $[s, B] \cap F=\emptyset$.

Notice that the $\sigma$-ideal $C R_{0}$ is defined on subsets of the set $[\omega]^{\omega}$ which can be identified with a subset of $2^{\omega}$ via characteristic functions. Thus, in the next part we deal with subsets of $2^{\omega}$.

Theorem 3. For any $[s, A]$, where $A \in[\omega]^{\omega}$, $\max s<\min A$, there exists a negligible set $H$ (even "small" in the sense of T. Bartoszyniski) such that

$$
\forall_{t \in 2^{\omega}} \exists_{B \in[A] \omega}[s, B] \subseteq H+t .
$$

Proof. Consider a partition of $\omega$ into finite disjoint intervals, say $\left(I_{n}\right)_{n<\omega}$, which satisfies the following conditions:

1.

$$
\forall_{n<\omega} \frac{\ln \left|A \cap I_{n}\right|+1}{\left|A \cap I_{n}\right|} \leq \frac{1}{2^{n}}
$$

2.

$$
\max (s)<\min \left(I_{0}\right) .
$$

By Lorentz's theorem (see for example [NSW]), we can find $H_{n} \subseteq 2^{I_{n}}$ with the properties:

1.

$$
\left|H_{n}\right| \leq \frac{\ln \left|A \cap I_{n}\right|+1}{\left|A \cap I_{n}\right|} \cdot 2^{\left|I_{n}\right|}
$$

2.

$$
H_{n}+\left\{e_{a}^{n}: a \in A \cap I_{n}\right\}=2^{I_{n}},
$$


where $e_{a}^{n}$ is an element of $2^{I_{n}}$ defined by the following condition:

$$
e_{a}^{n}(b)= \begin{cases}0 & \text { if } \quad a \neq b, \\ 1 & \text { if } \quad a=b .\end{cases}
$$

It is clear that the set

$$
H=\left\{x \in 2^{\omega}: \exists_{n}^{\infty} x \mid I_{n} \in H_{n}\right\}
$$

is negligible; moreover, it is "small" (see [BJ] for the definition of a "small" set). Let us fix $t \in 2^{\omega}$. For every $n \in \omega$, there exists $a_{n} \in A$ such that

$$
\left(t \mid I_{n}\right)+e_{a_{n}}^{n} \in H_{n}
$$

Put $B=\left\{a_{n}\right\}_{n<\omega}$. It is sufficient to show that $[s, B] \subseteq H+t$. So, let $C \in[\omega]^{\omega} \subseteq 2^{\omega}$ satisfy $s \subseteq C \subseteq s \cup B$. We have that

$$
\exists_{n}^{\infty} C \mid I_{n}=e_{a_{n}}^{n} .
$$

Thus,

$$
C \in\left\{x: \exists_{n}^{\infty}(x+t) \mid I_{n} \in H_{n}\right\}=H+t .
$$

Theorem 4. Every strongly meager set is a completely Ramsey null set.

Proof. Immediately follows from Theorem 3

In the proof of Theorem 11 we used an observation that for a strong measure zero set $X \subseteq 2^{\omega}$ and for every continuous function $f: 2^{\omega} \rightarrow 2^{\omega}$, the image $f[X]$ is also strongly measure zero. It is due to Rothberger (see [M]) that (assuming $\mathrm{CH}$ ) there exist a set $X$ of strong measure zero and a continuous function $f: X \rightarrow 2^{\omega}$ such that $f[X]=2^{\omega}$. Also, (assuming $\mathrm{CH}$ ) one can find a strongly meager set $X$ and a continuous function $f: X \rightarrow 2^{\omega}$ such that $f[X]=2^{\omega}$. It is a natural guess that for a strongly meager set $X$, and for every continuous function $f: 2^{\omega} \rightarrow 2^{\omega}$, we have that $f[X]$ is also strongly meager. However, it is not even known if for such $X$ and $f$, the image $f[X]$ has to be an $s_{0}$-set.

Question 1. Is it true that for a strongly meager set $X$ and for every continuous $f: 2^{\omega} \rightarrow 2^{\omega}, f[X]$ has the Marczewski property $s_{0}$ ?

Question 2. Is it possible to find for each continuous function $f: 2^{\omega} \rightarrow 2^{\omega}$ a negligible set $H$ such that

$$
\forall_{t \in 2^{\omega}} \exists_{P \in \text { Perf }} f^{-1}[P] \subseteq H+t ?
$$

We have the following simple observation.

Observation 1. A positive answer to Question 2 yields the answer "yes" to Question 1 .

Proof. Assume that $X$ is strongly meager and $f: 2^{\omega} \rightarrow 2^{\omega}$ is a continuous function. Let $P \subseteq 2^{\omega}$ be a perfect set. Fix a homeomorphism $h: P \rightarrow 2^{\omega}$ and a retraction $g: 2^{\omega} \rightarrow P$. Consider $\phi=h \circ g \circ f$. There is a negligible set $H \subseteq 2^{\omega}$ such that

$$
\forall_{t \in 2^{\omega}} \exists_{Q \subseteq 2^{\omega}, Q} \text { perfect } \phi^{-1}[Q] \subseteq H+t .
$$

Take $t_{0} \in 2^{\omega}$ such that $\left(H+t_{0}\right) \cap X=\emptyset$. We have that for some perfect set $Q^{\prime} \subseteq P$, $f^{-1}\left[Q^{\prime}\right] \cap X=\emptyset$. This implies that $f[X] \cap Q^{\prime}=\emptyset$. 
Theorem 5. For every continuous $f: 2^{\omega} \rightarrow 2^{\omega}$, there is $H$, a closed nowhere dense set, such that

$$
\forall_{t \in 2^{\omega}} \exists_{P \in \text { Perf }} f^{-1}[P] \subseteq H+t .
$$

Proof. Let $n \in \omega$. Choose $N_{n} \in \omega$ such that for every $s \in 2^{N_{n}}$, one can find $t_{s}^{(n)} \in 2^{n}$ satisfying

$$
f\left[C_{s}\right] \subseteq C_{t_{s}^{(n)}}
$$

where for $t \in 2^{<\omega}, C_{t}=\left\{x \in 2^{\omega}: x \mid\right.$ length $\left.(t)=t\right\}$.

We put $n_{0}=1, k_{0}=N_{n_{0}}$ and choose $n_{1}$ to get the inequality $2^{n_{1}-n_{0}}>2^{k_{0}}+1$, $k_{1}=N_{n_{1}}$. In general, we choose $n_{l}$ in such a way that the inequality $2^{n_{l}-n_{l-1}}>$ $2^{k_{l-1}}+1, k_{l}=N_{n_{l}}$, holds.

We define

and we put

$$
H_{l}=\left\{s \in 2^{\left[k_{l-1}, k_{l}\right)}: s \not \equiv 0 \mid\left[k_{l-1}, k_{l}\right)\right\},
$$

$$
H=\left\{x \in 2^{\omega}: \forall_{l>0} x \mid\left[k_{l-1}, k_{l}\right) \in H_{l}\right\} .
$$

One can check that $H \in \mathcal{M G R}$ (meager sets). In fact, $H$ is a closed, nowhere dense set. Consider $t \in 2^{\omega}$. We have that

$$
\begin{aligned}
& f\left[2^{\omega} \backslash\left\{x \in 2^{\omega}:(x+t)\left|\left[k_{l-1}, k_{l}\right) \not \equiv 0\right|\left[k_{l-1}, k_{l}\right)\right\}\right] \\
& \subseteq f\left[\left\{x \in 2^{\omega}: x\left|\left[k_{l-1}, k_{l}\right)=t\right|\left[k_{l-1}, k_{l}\right)\right\}\right]
\end{aligned}
$$

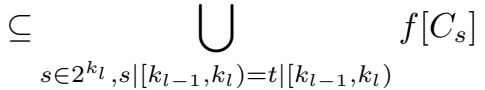

$$
\begin{aligned}
& \subseteq \bigcup_{s \in 2^{k_{l}}, s\left|\left[k_{l-1}, k_{l}\right)=t\right|\left[k_{l-1}, k_{l}\right)} C_{t_{s}^{\left(n_{l}\right)}} .
\end{aligned}
$$

The last inclusion follows from (1).

Since $2^{k_{l-1}}+1<2^{n_{l}-n_{l-1}}$, one can find $x_{l}^{(0)}, x_{l}^{(1)} \in 2^{\left[n_{l-1} ; n_{l}\right)}, x_{l}^{(0)} \neq x_{l}^{(1)}$, such that

$$
\begin{gathered}
\left\{x \in 2^{\omega}: x \mid\left[n_{l-1}, n_{l}\right)=x_{l}^{(r)}\right\} \\
\cap f\left[2^{\omega} \backslash\left\{x \in 2^{\omega}:(x+t)\left|\left[k_{l-1}, k_{l}\right) \not \equiv 0\right|\left[k_{l-1}, k_{l}\right)\right\}\right]=\emptyset,
\end{gathered}
$$

for $r \in 2$.

We put

$$
P=\left\{x \in 2^{\omega}: \forall_{l>0} x \mid\left[n_{l-1}, n_{l}\right) \in\left\{x_{l}^{(0)}, x_{l}^{(1)}\right\}\right\} .
$$

Clearly, $P$ is a perfect subset of $2^{\omega}$. We must check that

$$
f^{-1}[P] \subseteq H+t .
$$

It suffices to show that if $l<\omega$ and $z \in 2^{\omega}$ are such that

$$
f(z) \mid\left(\left[n_{l-1}, n_{l}\right)\right) \in\left\{x_{l}^{(0)}, x_{l}^{(1)}\right\}
$$

then

$$
(z+t)\left|\left[k_{l-1}, k_{l}\right) \not \equiv 0\right|\left[k_{l-1}, k_{l}\right)
$$

So, take $z \in 2^{\omega}$ satisfying

$$
(z+t)\left|\left[k_{l-1}, k_{l}\right) \equiv 0\right|\left[k_{l-1}, k_{l}\right)
$$

This means that

$$
z \in 2^{\omega} \backslash\left\{x \in 2^{\omega}:(x+t)\left|\left[k_{l-1}, k_{l}\right) \not \equiv 0\right|\left[k_{l-1}, k_{l}\right)\right\} .
$$

Thus, $f(z) \mid\left[n_{l-1}, n_{l}\right) \notin\left\{x_{l}^{(0)}, x_{l}^{(1)}\right\}$ by (2). 


\section{REFERENCES}

[BJ] H. Judah, T. Bartoszyński, Set theory, A.K. Peters, Wellesley, Massachusetts, 1995. MR 96k:03002

[BRR] L. Bukovský, I. Recław and M. Repický, Spaces not distinguishing pointwise and quasinormal convergence of real functions, Topology and its Applications 41 (1991), 25-40. MR 93b:54037

[M] A.W. Miller, Special subsets of the real line in 'Handbook of set - theoretic topology', (1984b), 201 - 233, North-Holland, Amsterdam-New York. MR 86i:54037

[N] A. Nowik, Remarks about a transitive version of perfectly meager sets, Real Analysis Exchange 22(1) (1996/97), 406 - 412. MR 97m:54043

[NSW] A. Nowik, M. Scheepers, T. Weiss, The algebraic sum of sets of real numbers with strong measure zero sets. Journal of Symbolic Logic 63 (1998), 301 - 324. MR 99c:54049

[P] J. Pawlikowski, All Sierpiński sets are strongly meager, 1992, Arch. Mat. Logic 35 (1996) 281 - 285. MR 98i:03066

[S] M. Scheepers, Additive properties of sets of real numbers and an infinite game, Quaestiones Mathematicae 16 (1993), 177 - 191. MR 94e:04003

Institute of Mathematics, University of Gdańsk, Ul. Wita Stwosza 57, 80 - 952 Gdańsk, Poland

E-mail address: matan@paula.univ.gda.pl

Institute of Mathematics, WSRP, 08-110 Siedlce, Poland

E-mail address: weiss@wsrp.siedlce.pl 